\title{
Polynomial set-valued functions
}

\author{
by JoAnna SzcZawińska (Kraków)
}

\begin{abstract}
The aim of this paper is to give a necessary and sufficient condition for a set-valued function to be a polynomial s.v. function of order at most 2 .

Let $X, Z$ be vector spaces over $\mathbb{Q}$ and $C$ be a $\mathbb{Q}$-convex subset of $X$. Let $f: C \rightarrow Z$ be an arbitrary function and $h \in X$. The difference operator $\Delta_{h}$ is given by the formula
\end{abstract}

$$
\Delta_{h} f(x):=f(x+h)-f(x)
$$

for $x \in C$ such that $x+h \in C$. The iterates $\Delta_{h}^{n}$ of $\Delta_{h}$ are given by the recurrence

$$
\Delta_{h}^{0} f:=f, \quad \Delta_{h}^{n+1} f:=\Delta_{h}\left(\Delta_{h}^{n} f\right), \quad n=0,1,2, \ldots
$$

The expression $\Delta_{h}^{n} f$ is a function defined for all $x \in C$ such that $x+n h \in C$. It is easy to see that $x+k h \in C$ for $k=1, \ldots, n-1$ whenever $x, x+n h \in C$.

A function $f: C \rightarrow Z$ is said to be a Jensen function if it satisfies the Jensen functional equation

$$
f\left(\frac{x+y}{2}\right)=\frac{1}{2}[f(x)+f(y)]
$$

for all $x, y \in C$. if

A function $f: C \rightarrow Z$ is called a polynomial function of order at most $n$

$$
\Delta_{h}^{n+1} f(x)=0
$$

for every $x \in C$ and $h \in X$ such that $x+(n+1) h \in C$.

We have

$$
\Delta_{h}^{n} f(x)=\sum_{k=0}^{n}(-1)^{n-k}\left(\begin{array}{l}
n \\
k
\end{array}\right) f(x+k h)
$$

1991 Mathematics Subject Classification: 54C60, 26E25, 39A70.

Key words and phrases: polynomial set-valued functions, difference operators, biadditive functions, Jensen function. 
for $n \in \mathbb{N}, h \in X$ and $x \in C$ such that $x+n h \in C$ (see e.g. [3], Corollary 2, p. 368).

R. Ger has proved that every polynomial function $f: C \rightarrow Z$ of order at most $n$ admits an extension to a polynomial function of order at most $n$ on the whole $X$ (see Theorem 2 of [2]). Therefore, due to Theorem 3 of [3] (p. 379), we can formulate the following theorem:

TheOREm 1. Let $X, Z$ be $\mathbb{Q}$-linear spaces and $C$ be a nonempty $\mathbb{Q}$-convex subset of $X$. If $f: C \rightarrow Z$ is a polynomial function of order at most $n$ then

$$
\Delta_{h_{1} \ldots h_{n+1}} f(x):=\Delta_{h_{1}} \Delta_{h_{2}} \ldots \Delta_{h_{n+1}} f(x)=0
$$

for $x \in C$ and $h_{1}, \ldots, h_{n+1} \in X$ such that $x+\varepsilon_{1} h_{1}+\ldots+\varepsilon_{n+1} h_{n+1} \in C$, $\varepsilon_{1}, \ldots, \varepsilon_{n+1} \in\{0,1\}$.

We are going to deal with polynomial set-valued functions (abbreviated to s.v. functions in the sequel). Let $Y$ be a real Hausdorff topological vector space. The symbol $n(Y)$ will stand for the set of all non-empty subsets of $Y$. The set of all convex and compact members of $n(Y)$ will be denoted by $c c(Y)$.

Rådström's equivalence relation $\sim$ (see [5]) is defined on $(c c(Y))^{2}$ by stating $(A, B) \sim(C, D)$ if $A+D=B+C$. The equivalence class containing $(A, B)$ is denoted by $[A, B]$. The quotient space $\mathcal{Z}=(c c(Y))^{2} / \sim$, with addition defined by

$$
[A, B]+[D, E]:=[A+D, B+E],
$$

and scalar multiplication

$$
\lambda[A, B]:= \begin{cases}{[\lambda A, \lambda B],} & \lambda \geq 0, \\ {[-\lambda B,-\lambda A],} & \lambda<0,\end{cases}
$$

is a real vector space.

The following result of Rådström (see [5], Lemma 3) is useful.

Lemma 1. Let $A, B$ be convex and closed sets in $Y$ and let $C$ be nonempty and bounded. Then $A+C=B+C$ implies $A=B$.

Let $Y$ be a topological vector space and let $\mathcal{W}$ be a base of neighbourhoods of zero in $Y$. The space $n(Y)$ may be considered as a topological space with the Hausdorff topology. In this topology the families of sets

$$
N_{W}(A):=\{B \in n(Y): A \subseteq B+W \text { and } B \subseteq A+W\},
$$

where $W$ runs over the base $\mathcal{W}$, form a base of neighbourhoods of the set $A \in n(Y)$ (see $[6])$.

The three lemmas below can be found e.g. in [4] (Lemmas 5.6 and 3.2).

Lemma 2. Let $Y$ be a topological vector space and $A_{n}, B_{n}, A, B \in n(Y)$ for $n \in \mathbb{N}$. If $A_{n} \rightarrow A$ and $B_{n} \rightarrow B$ (in the Hausdorff topology on $n(Y)$ ), 
then $A_{n}+B_{n} \rightarrow A+B$. If $A$ is bounded, then the function $t \rightarrow t A$ is continuous.

Lemma 3. If $\lambda_{n} \rightarrow 0$ and $A \in n(Y)$ is bounded, then $\lambda_{n} A \rightarrow\{0\}$.

Lemma 4. If $\lambda_{n} \rightarrow \lambda_{0}$ and $A \in n(Y)$ is bounded, then $\lambda_{n} A \rightarrow \lambda_{0} A$.

The next lemma is proved in [1] for a metric space $Y$.

Lemma 5. Let $Y$ be a topological vector space. If $A_{n} \rightarrow A$ (in the Hausdorff topology on $n(Y))$ and $A$ is closed then $A=\bigcap_{n=1}^{\infty} \overline{\bigcup_{m \geq n} A_{m}}$.

Proof. Fix $n \in \mathbb{N}$ and $W \in \mathcal{W}$, where $\mathcal{W}$ denotes a base of neighbourhoods of zero in $Y$. Since $A_{n} \rightarrow A$, there is $n_{0} \in \mathbb{N}$ such that $A \subseteq A_{m}+W$ for every $m \geq n_{0}$. Hence, $A \subseteq \bigcup_{m \geq n} A_{m}+W$ for $W \in \mathcal{W}$. Therefore, we have

$$
A \subseteq \bigcap_{n=1}^{\infty} \overline{\bigcup_{m \geq n} A_{m}}
$$

Now, fix $W \in \mathcal{W}$. Let $V \in \mathcal{W}$ with $V+V \subseteq W$. There is $n_{0} \in \mathbb{N}$ such that if $n \geq n_{0}$, then

$$
A_{n} \subseteq A+V .
$$

Choose an $x \in \bigcap_{n=1}^{\infty} \overline{\bigcup_{m \geq n} A_{m}}$. Hence $x \in A_{m}+V$ for some $m \geq n_{0}$. Then by (1),

$$
x \in A+V+V \subseteq A+W,
$$

that is, $x \in \bar{A}=A$. Consequently, $\bigcap_{n=1}^{\infty} \overline{\bigcup_{m \geq n} A_{m}} \subseteq A$.

LEMMA 6. Let $Y$ be a topological vector space and $A_{n}, B, C \in c c(Y)$ for $n \in \mathbb{N}$. If $A_{n}+B=: C_{n} \rightarrow C$, then there exists $A \in c c(Y)$ such that $C=A+B$.

Proof. By the last lemma, Lemma 5.3 of [4] and the fact that the algebraic sum of a compact set and a closed set is closed, we have

$$
\begin{aligned}
C & =\bigcap_{n=1}^{\infty} \overline{\bigcup_{m \geq n}\left(A_{m}+B\right)}=\bigcap_{n=1}^{\infty} \overline{\bigcup_{m \geq n} A_{m}+B}=\bigcap_{n=1}^{\infty} \overline{\overline{\bigcup_{m \geq n} A_{m}}+B} \\
& =\bigcap_{n=1}^{\infty}\left(\overline{\bigcup_{m \geq n} A_{m}}+B\right)=\bigcap_{n=1}^{\infty} \overline{\bigcup_{m \geq n} A_{m}}+B .
\end{aligned}
$$

Hence

$$
\begin{aligned}
C & =\operatorname{clconv}\left(\bigcap_{n=1}^{\infty} \overline{\bigcup_{m \geq n} A_{m}}+B\right)=\operatorname{cl}\left(\operatorname{conv} \bigcap_{n=1}^{\infty} \overline{\bigcup_{m \geq n} A_{m}}+B\right) \\
& =\operatorname{cl}\left(\operatorname{clconv} \bigcap_{n=1}^{\infty} \overline{\bigcup_{m \geq n} A_{m}}+B\right)=\operatorname{clconv} \bigcap_{n=1}^{\infty} \overline{\bigcup_{m \geq n} A_{m}}+B .
\end{aligned}
$$


Put

$$
A:=\operatorname{clconv} \bigcap_{n=1}^{\infty} \overline{\bigcup_{m \geq n} A_{m}} .
$$

The set $A$ is of course closed and convex and $A+B=C$. Since $A \subset C-B$, $A$ is compact.

Definition 1. A set $S \subseteq X$ is said to be a $\mathbb{Q}$-convex cone if $S+S \subseteq S$ and $\lambda S \subseteq S$ for all $\lambda \in \mathbb{Q} \cap\langle 0, \infty)$.

Now consider an s.v. function $F: S \rightarrow c c(Y)$, where $S \subseteq X$ denotes a $\mathbb{Q}$-convex cone. Define $f: S \rightarrow \mathcal{Z}$ as follows:

$$
f(x):=[F(x),\{0\}] .
$$

Definition 2. Let $h \in X$. The difference operator of the function $f$ : $S \rightarrow \mathcal{Z}$ given by (2) is called the difference operator of the s.v. function $F$, i.e. $\Delta_{h} F(x):=\Delta_{h} f(x)=[F(x+h), F(x)]$ for $x \in S$ and $h \in X$ such that $x+h \in S$, and $\Delta_{h}^{n} F(x):=\Delta_{h}^{n} f(x)$ for $x \in S$ and $h \in X$ such that $x+n h \in S$.

Definition 3. An s.v. function $F: S \rightarrow c c(Y)$ is called a polynomial s.v. function of order at most $n$ if the function $f: S \rightarrow \mathcal{Z}$ given by (2) is a polynomial function of order at most n, i.e. $\Delta_{h}^{n+1} F(x)=0$ for $x \in S$ and $h \in X$ such that $x+(n+1) h \in S$.

Observe that if $F: S \rightarrow c c(Y)$ is polynomial of order 0, i.e. $\Delta_{h} F(x)=0$ for $x \in S$ and $h \in X$ such that $x+h \in S$, then $F$ is constant.

Now, let $F$ be a polynomial s.v. function of order at most one. Then

$$
\Delta_{h}^{2} F(x)=[F(x+2 h)+F(x), 2 F(x+h)]=0
$$

for $x \in S$ and $h \in X$ such that $x+2 h \in S$. This means that

$$
F(x+2 h)+F(x)=2 F(x+h)
$$

for $x \in S$ and $h \in X$ such that $x+2 h \in S$.

Putting $h:=(y-x) / 2 \in X$ in (3), where $x, y$ are arbitrary from $S$, we get $x+h=(x+y) / 2 \in S, x+2 h=y \in S$ and

$$
F(y)+F(x)=2 F\left(\frac{x+y}{2}\right), \quad x, y \in S .
$$

So, if $\Delta_{h}^{2} F(x)=0$, then $F$ satisfies the Jensen equation (4). Conversely, if $F$ satisfies the above equation, then $F$ is a polynomial function of order at most one.

If $F$ is a polynomial s.v. function of order at most one then the function $g: S \rightarrow \mathcal{Z}$ given by

$$
g(x):=\Delta_{x} f(0)=[F(x), F(0)]
$$


is additive. Indeed, by Lemma 3 (p. 367) of [3] and Theorem 1,

$$
g(x+y)=\Delta_{x+y} f(0)=\Delta_{x, y} f(0)+\Delta_{x} f(0)+\Delta_{y} f(0)=g(x)+g(y)
$$

for all $x, y \in S$. Then $g(n x)=n g(x)$ for all $n \in \mathbb{N}$ and $x \in S$, which gives

$$
[F(n x), F(0)]=n[F(x), F(0)] .
$$

Hence

$$
\frac{1}{n} F(n x)+F(0)=\frac{1}{n} F(0)+F(x)
$$

for $x \in S$ and $n \in \mathbb{N}$. Since the limit of the right-hand side exists, so does the limit of the left-hand side. By Lemma 6 , there is a set $A(x) \in c c(Y)$ such that

$$
A(x)+F(0)=\lim _{n \rightarrow \infty}\left(\frac{1}{n} F(0)+F(x)\right)=F(x)
$$

for $x \in S$. It follows that

$$
[A(x),\{0\}]=[F(x), F(0)],
$$

so the s.v. function $A$ is additive. Conversely, if $A: S \rightarrow c c(Y)$ is additive and $F(0) \in c c(Y)$, then the s.v. function $F$ given by $F(x)=F(0)+A(x)$ is a polynomial s.v. function of order at most one. By the above considerations we can formulate a theorem proved by K. Nikodem [4] in a different way.

Theorem 2. Let $X$ be a real vector space, $S$ be a $\mathbb{Q}$-convex cone in $X$ and let $Y$ be a real topological vector space. Then $F: S \rightarrow c c(Y)$ is a polynomial s.v. function of order at most one if and only if there exists an additive s.v. function $A: S \rightarrow c c(Y)$ such that $F(x)=F(0)+A(x)$ for $x \in S$.

An s.v. function $F$ is a polynomial function of order 2 if and only if

$$
F(x+3 h)+3 F(x+h)=3 F(x+2 h)+F(x)
$$

for $x \in S$ and $h \in X$ such that $x+3 h \in S$. It is easily seen that if

$$
F(x)=A_{0}+A_{1}(x)+A_{2}(x)
$$

for $x \in S$, where $A_{0} \in c c(Y), A_{1}, A_{2}: S \rightarrow c c(Y), A_{1}$ is additive and $A_{2}$ is the diagonalization of a biadditive s.v. function $\bar{A}_{2}: S \times S \rightarrow c c(Y)$ (i.e. $\left.A_{2}(x)=\bar{A}_{2}(x, x), x \in S\right)$ then $F$ is a polynomial s.v. function of order at most 2 .

Now, let us consider an example. Let $S=\langle 0, \infty)$ and $F: S \rightarrow c c(\mathbb{R})$ be given by the formula $F(x):=\left\langle 2 x, x^{2}+1\right\rangle, x \in S$. Obviously, $F$ is a polynomial function of order at most 2 but we cannot present it in the form (5). In fact, putting $x=0$ in (5), we get $A_{0}=\langle 0,1\rangle$. Next, putting $x=1$ in (5), we obtain

$$
\langle 0,1\rangle+A_{1}(1)+A_{2}(1)=\langle 2,2\rangle,
$$

which is not possible. 
Remark 1 . Let $F: S \rightarrow c c(Y)$ be a polynomial s.v. function of order at most 2 and let $\bar{f}: X \rightarrow \mathcal{Z}$ denote an extension of the function $f$ defined by (2). The function $g: X \times X \rightarrow \mathcal{Z}$ given by

$$
g(x, y):=\frac{1}{2} \Delta_{x, y} \bar{f}(0)
$$

is biadditive and

(6) $g(x, y)=\frac{1}{2} \Delta_{x, y} f(0)=\frac{1}{2}[F(x+y)+F(0), F(x)+F(y)] \quad$ for $x, y \in S$.

Proof. A polynomial extension $\bar{f}: X \rightarrow \mathcal{Z}$ of order at most 2 of the function $f$ exists in view of Theorem 2 of [2]. Note that $g$ is symmetric. Fix $x, y, z \in X$. By Lemma 3 of [3] (p. 367) and Theorem 1 we have

$$
\begin{aligned}
g(x+z, y) & =\frac{1}{2} \Delta_{x+z, y} f(0)=\frac{1}{2} \Delta_{y} \Delta_{x+z} f(0) \\
& =\frac{1}{2} \Delta_{y}\left(\Delta_{x, z} f(0)+\Delta_{x} f(0)+\Delta_{z} f(0)\right) \\
& =\frac{1}{2} \Delta_{x, y, z} f(0)+\frac{1}{2} \Delta_{x, y} f(0)+\frac{1}{2} \Delta_{z, y} f(x)(0) \\
& =g(x, y)+g(z, y) .
\end{aligned}
$$

By (2), the equation (6) is obvious.

THEOREM 3. Let $F: S \rightarrow c c(Y)$ be a polynomial function of order at most 2. Then there exists a polynomial s.v. function $A: S \rightarrow c c(Y)$ of order at most 2 such that

$$
\begin{aligned}
& \frac{1}{2} F(0)+\frac{1}{2} F(2 x)=A(x)+F(x), \quad x \in S, \\
& A(\lambda x)=\lambda^{2} A(x), \quad x \in S, \quad \lambda \in \mathbb{Q} \cap\langle 0, \infty),
\end{aligned}
$$

and the function

$$
x \rightarrow[F(x), F(0)+A(x)], \quad x \in S,
$$

is additive.

Proof. By Remark 1 the function $g: X \times X \rightarrow \mathcal{Z}$ given by $g(x, y):=$ $\frac{1}{2} \Delta_{x, y} \bar{f}(0)$ is biadditive, where $\bar{f}$ denotes an extension of $f$.

First, we prove that

$$
\begin{aligned}
F\left(\sum_{k=1}^{n} x_{k}\right)+(n-2) & \sum_{k=1}^{n} F\left(x_{k}\right) \\
& =\frac{(n-2)(n-1)}{2} F(0)+\sum_{1 \leq k<l \leq n} F\left(x_{k}+x_{l}\right),
\end{aligned}
$$

where $n \geq 2$ and $x_{1}, \ldots, x_{n} \in S$. If $n=2$, then (7) is trivial. Now, assume that $(7)$ holds for $n \geq 2$. Let $x_{1}, \ldots, x_{n+1} \in S$. Since

$$
g\left(\sum_{k=1}^{n} x_{k}, x_{n+1}\right)=\sum_{k=1}^{n} g\left(x_{k}, x_{n+1}\right),
$$


we have

$$
\begin{aligned}
{\left[F\left(\sum_{k=1}^{n} x_{k}+x_{n+1}\right)+\right.} & \left.F(0), F\left(\sum_{k=1}^{n} x_{k}\right)+F\left(x_{n+1}\right)\right] \\
& =\sum_{k=1}^{n}\left[F\left(x_{k}+x_{n+1}\right)+F(0), F\left(x_{k}\right)+F\left(x_{n+1}\right)\right],
\end{aligned}
$$

whence

$$
\begin{aligned}
F\left(\sum_{k=1}^{n+1} x_{k}\right)+ & F(0)+\sum_{k=1}^{n} F\left(x_{k}\right)+n F\left(x_{n+1}\right) \\
& =F\left(\sum_{k=1}^{n} x_{k}\right)+F\left(x_{n+1}\right)+\sum_{k=1}^{n} F\left(x_{k}+x_{n+1}\right)+n F(0) .
\end{aligned}
$$

By the Rådström lemma

$$
\begin{aligned}
F\left(\sum_{k=1}^{n+1} x_{k}\right)+\sum_{k=1}^{n} F\left(x_{k}\right)+(n-1) F\left(x_{n+1}\right) \\
=F\left(\sum_{k=1}^{n} x_{k}\right)+\sum_{k=1}^{n} F\left(x_{k}+x_{n+1}\right)+(n-1) F(0) .
\end{aligned}
$$

Hence and by the induction hypothesis we have

$$
\begin{aligned}
F\left(\sum_{k=1}^{n+1} x_{k}\right)+(n-1) \sum_{k=1}^{n+1} F\left(x_{k}\right) \\
=F\left(\sum_{k=1}^{n} x_{k}\right)+(n-2) \sum_{k=1}^{n} F\left(x_{k}\right)+\sum_{k=1}^{n} F\left(x_{k}+x_{n+1}\right)+(n-1) F(0) \\
=\frac{(n-2)(n-1)}{2} F(0)+\sum_{1 \leq k<l \leq n} F\left(x_{k}+x_{l}\right) \\
\quad+\sum_{k=1}^{n} F\left(x_{k}+x_{n+1}\right)+(n-1) F(0) \\
=\sum_{1 \leq k<l \leq n+1} F\left(x_{k}+x_{l}\right)+\frac{(n-1) n}{2} F(0)
\end{aligned}
$$

which ends the induction.

Putting $x=x_{1}=\ldots=x_{n}$ in (7), we have

$$
F(n x)+n(n-2) F(x)=\left(\begin{array}{c}
n-1 \\
2
\end{array}\right) F(0)+\left(\begin{array}{l}
n \\
2
\end{array}\right) F(2 x), \quad n \geq 3, x \in S,
$$


and

(8) $\frac{F(n x)}{n(n-2)}+F(x)=\frac{\left(\begin{array}{c}n-1 \\ 2\end{array}\right)}{n(n-2)} F(0)+\frac{\left(\begin{array}{l}n \\ 2\end{array}\right)}{n(n-2)} F(2 x), \quad n \geq 3$.

By Lemmas 4 and 2 the limit of the right-hand side of (8) exists; consequently, so does the limit of the left-hand side, and by Lemma 6 , for all $x \in S$, there is a set $A(x) \in c c(Y)$ such that

$$
\frac{1}{2} F(0)+\frac{1}{2} F(2 x)=A(x)+F(x), \quad x \in S .
$$

This means that

$$
[A(x),\{0\}]=\frac{1}{2}[F(2 x)+F(0), 2 F(x)]=g(x, x), \quad x \in S .
$$

Therefore, the function $a: X \rightarrow \mathcal{Z}$ defined by $a(x):=g(x, x)$ is the diagonalization of the biadditive function $g$ and

$$
a(x)=[A(x),\{0\}] \quad \text { for } x \in S .
$$

By Definition 3, $A$ is a polynomial function of order at most 2. Since $g$ is biadditive, for $x \in S$ and $\lambda \in \mathbb{Q} \cap\langle 0, \infty)$,

$$
[A(\lambda x),\{0\}]=g(\lambda x, \lambda x)=\lambda^{2} g(x, x)=\lambda^{2}[A(x),\{0\}],
$$

which means that $A(\lambda x)=\lambda^{2} A(x)$.

Finally, observe that the function $x \rightarrow f(x)-a(x), x \in S$, is a Jensen function. Indeed, let $x \in S$ and $h \in X$ with $x+2 h \in S$. Then

$\Delta_{h}^{2}(f(x)-a(x))=\Delta_{h}^{2} f(x)-2 g(h, h)=\Delta_{h}^{2} f(x)-\Delta_{h}^{2} f(0)=\Delta_{h}^{2} \Delta_{x} f(0)=0$, by Theorem 1 and biadditivity of $g$. Define $\bar{g}: S \rightarrow \mathcal{Z}$ by

$$
\bar{g}(x)=f(x)-a(x)-[F(0),\{0\}]=[F(x), A(x)+F(0)] .
$$

Then the considerations above and the fact that $\bar{g}(0)=0$ imply the additivity of $\bar{g}$.

Definition 4 (cf. [3]). Let $S$ be a convex cone in a vector space $X$ over $\mathbb{Q}$. A set $\mathcal{E}$ is called a base of $S$ if $\mathcal{E}$ is linearly independent and the cone is spanned by $\mathcal{E}$, i.e., the set

$$
\left\{x \in X: x=\sum_{k=1}^{n} \lambda_{k} e_{k}, \quad e_{1}, \ldots, e_{n} \in \mathcal{E}, \lambda_{1}, \ldots, \lambda_{n} \in \mathbb{Q} \cap\langle 0, \infty), n \in \mathbb{N}\right\}
$$

coincides with $S$.

TheOREM 4. Let $X$ be a vector space over $\mathbb{Q}$ and $Y$ be a topological vector space, and let $S \subseteq X$ be a cone with a base. Then $F: S \rightarrow c c(Y)$ is a polynomial s.v. function of order at most 2 if and only if there exist additive s.v. functions $\bar{B}, \bar{C}: S \rightarrow c c(Y)$ and biadditive s.v. functions $\bar{D}, \bar{H}: S \times S \rightarrow$ $c c(Y)$ such that 
(9)

$$
F(x)+\bar{C}(x)+\bar{H}(x, x)=F(0)+\bar{D}(x, x)+\bar{B}(x)
$$

for $x \in S$.

Proof. Since a cone with a base is $\mathbb{Q}$-convex, by Theorem 3 there is an s.v. function $A: S \rightarrow c c(Y)$ such that

$$
x \rightarrow[F(x), F(0)+A(x)], \quad x \in S,
$$

is additive. There exist (see Theorem 1 of [7]) additive s.v. functions $\bar{B}, \bar{C}$ : $S \rightarrow c c(Y)$ such that

$$
[F(x), F(0)+A(x)]=[\bar{B}(x), \bar{C}(x)], \quad x \in S,
$$

which gives

$$
F(x)+\bar{C}(x)=F(0)+\bar{B}(x)+A(x), \quad x \in S .
$$

In view of Remark 1 ,

$$
g(x, y)=\frac{1}{2}[F(x+y)+F(0), F(x)+F(y)]
$$

is biadditive. Set

$$
D(x, y):=\frac{1}{2}(F(x+y)+F(0)), \quad H(x, y):=\frac{1}{2}(F(x)+F(y)),
$$

and let $\mathcal{E}$ be a base of $S$. Fix $x, y \in S$. There exist $n \in \mathbb{N}, \lambda_{1}, \ldots, \lambda_{n} \in$ $\mathbb{Q} \cap\langle 0, \infty)$ and $e_{1}, \ldots, e_{n} \in \mathcal{E}$ such that $x=\sum_{i=1}^{n} \lambda_{i} e_{i}$, and

$$
D(x, y)+\sum_{i=1}^{n} \lambda_{i} H\left(e_{i}, y\right)=H(x, y)+\sum_{i=1}^{n} \lambda_{i} D\left(e_{i}, y\right) .
$$

Similarly

$$
H\left(e_{i}, y\right)+\sum_{j=1}^{m} \mu_{j} D\left(e_{i}, \bar{e}_{j}\right)=D\left(e_{i}, y\right)+\sum_{j=1}^{m} \mu_{j} H\left(e_{i}, \bar{e}_{j}\right),
$$

where $y=\sum_{j=1}^{m} \mu_{j} \bar{e}_{j}, \bar{e}_{1}, \ldots, \bar{e}_{m} \in \mathcal{E}$ and $\mu_{1}, \ldots, \mu_{m} \in \mathbb{Q} \cap\langle 0, \infty)$. Hence

$$
\begin{aligned}
D(x, y)+\sum_{i=1}^{n} \lambda_{i} D\left(e_{i}, y\right)+\sum_{i=1}^{n} \sum_{j=1}^{m} \lambda_{i} \mu_{j} H\left(e_{i}, \bar{e}_{j}\right) \\
=D(x, y)+\sum_{i=1}^{n} \lambda_{i}\left[D\left(e_{i}, y\right)+\sum_{j=1}^{m} \mu_{j} H\left(e_{i}, \bar{e}_{j}\right)\right] \\
=D(x, y)+\sum_{i=1}^{n} \lambda_{i}\left[H\left(e_{i}, y\right)+\sum_{j=1}^{m} \mu_{j} D\left(e_{i}, \bar{e}_{j}\right)\right] \\
=D(x, y)+\sum_{i=1}^{n} \lambda_{i} H\left(e_{i}, y\right)+\sum_{i=1}^{n} \sum_{j=1}^{m} \lambda_{i} \mu_{j} D\left(e_{i}, \bar{e}_{j}\right) .
\end{aligned}
$$


Define

$$
\bar{D}(x, y):=\sum_{i=1}^{n} \sum_{j=1}^{m} \lambda_{i} \mu_{j} D\left(e_{i}, \bar{e}_{j}\right), \quad \bar{H}(x, y):=\sum_{i=1}^{n} \sum_{j=1}^{m} \lambda_{i} \mu_{j} H\left(e_{i}, \bar{e}_{j}\right),
$$

where $x=\sum_{i=1}^{n} \lambda_{i} e_{i}, y=\sum_{j=1}^{m} \mu_{j} \bar{e}_{j}, x, y \in S$. It is clear that $\bar{D}$ and $\bar{H}$ are biadditive and

$$
\frac{1}{2} F(x+y)+\frac{1}{2} F(0)+\bar{H}(x, y)=\frac{1}{2} F(x)+\frac{1}{2} F(y)+\bar{D}(x, y) .
$$

Setting $y=x$, we have

$$
\frac{1}{2} F(2 x)+\frac{1}{2} F(0)+\bar{H}(x, x)=F(x)+\bar{D}(x, x) .
$$

Hence and by Theorem 3,

$$
A(x)+\bar{H}(x, x)=\bar{D}(x, x)
$$

and by (10),

$$
\begin{aligned}
F(x)+\bar{C}(x)+\bar{H}(x, x) & =F(0)+\bar{B}(x)+A(x)+\bar{H}(x, x) \\
& =F(0)+\bar{D}(x, x)+\bar{B}(x), \quad x \in S .
\end{aligned}
$$

Thus (9) holds true. To end the proof it suffices to prove that $F$ is a polynomial s.v. function of order at most 2 if (9) is satisfied. By (9),

$$
\begin{aligned}
\Delta_{h}^{3} F(x) & \\
= & {[F(x+3 h)+3 F(x+h), 3 F(x+2 h)+F(x)] } \\
= & {[\bar{D}(x+3 h, x+3 h)+\bar{B}(x+3 h)+3 \bar{D}(x+h, x+h)+3 \bar{B}(x+h),} \\
& \quad \bar{H}(x+3 h, x+3 h)+\bar{C}(x+3 h)+3 \bar{H}(x+h, x+h)+3 \bar{C}(x+h)] \\
& \quad-[3 \bar{D}(x+2 h, x+2 h)+3 \bar{B}(x+2 h)+\bar{D}(x, x)+\bar{B}(x), \\
& 3 \bar{H}(x+2 h, x+2 h)+3 \bar{C}(x+2 h)+\bar{H}(x, x)+\bar{C}(x)] \\
= & {[\bar{D}(x+3 h, x+3 h)+3 \bar{D}(x+h, x+h), 3 \bar{D}(x+2 h, x+2 h)+\bar{D}(x, x)] } \\
= & {[\bar{H}(x+3 h, x+3 h)+3 \bar{H}(x+h, x+h), 3 \bar{H}(x+2 h, x+2 h)+\bar{H}(x, x)] } \\
& +[\bar{B}(x+3 h)+3 \bar{B}(x+h), 3 \bar{B}(x+2 h)+\bar{B}(x)] \\
& +[\bar{C}(x+3 h)+3 \bar{C}(x+h), 3 \bar{C}(x+2 h)+\bar{C}(x)] \\
= & \Delta_{h}^{3} \bar{D}(x, x)-\Delta_{h}^{3} \bar{H}(x, x)+\Delta_{h}^{3} \bar{B}(x)-\Delta_{h}^{3} \bar{C}(x)=0,
\end{aligned}
$$

for $x \in S$ and $h \in X$ such that $x+3 h \in S$, because $\bar{D}$ and $\bar{H}$ are biadditive and $\bar{B} y$ and $\bar{C}$ are additive. So, the proof is complete. 


\section{References}

[1] C. Castaing and M. Valadier, Convex Analysis and Measurable Multifunctions, Springer, Berlin, 1977.

[2] R. Ger, On extensions of polynomial functions, Results Math. 26 (1994), 281-289.

[3] M. Kuczma, An Introduction to the Theory of Functional Equations and Inequalities, PWN and Uniw. Śl., Warszawa-Kraków-Katowice, 1985.

[4] K. Nikodem, K-convex and K-concave set valued functions, Zeszyty Naukowe Politech. Łódzkiej, Mat. 559, Rozprawy Naukowe 114, 1989.

[5] H. Rådström, An embedding theorem for spaces of convex sets, Proc. Amer. Math. Soc. 3 (1952), 165-169.

[6] - One-parameter semigroups of subsets of a real linear space, Ark. Mat. 4 (1960), 87-97.

[7] A. Smajdor, On a functional equation, Ann. Math. Sil. 8 (1994), 217-226.

Institute of Mathematics

Pedagogical University

Podchorążych 2

30-084 Kraków, Poland

E-mail: smwilk@cyf-kr.edu.pl

Reçu par la Rédaction le 18.5.1995

Révisé le 7.6.1996 